\title{
BMC Bioinformatics reviewer acknowledgement 2015
}

Dirk Krüger

\section{Contributing reviewers}

The editors of BMC Bioinformatics would like to thank all our reviewers who have contributed to the journal in Volume 16 (2015).

Suyu Mei

China

Alexey Goltsov

UK

Andrzej Kierzek

UK

Adam P Levine

UK

Alan Wee-Chung Liew

Australia

Andrew Wood

UK

Andrej-Nikolai Spiess

Germany

Andrej Spiess

Germany

Aalt Van Dijk

Netherlands

Aaron Shafer

Sweden

Andreas Bender

UK

Alexej Abyzov

USA

Andrew C Doxey

Canada
Ariel Chernomoretz

Argentina

Alice Cleynen

USA

Andrew Cohen

USA

Anthony Cox

UK

Adrian Altenhoff

Switzerland

Alexandre Dufour

France

Ana Rita Grosso

Portugal

Abhinav Grover

India

Alexander Gusev

USA

Andrew Hart

Chile

Kamesh Arumugam

USA

Alla Karnovsky

USA

Andriy Kryshtafovych

USA

\section{Alain Hauser \\ Switzerland}

Alba Cristina De Melo

Brazil

Aleksey Zimin

USA

Alexander Dobrovic

Australia

Alex Valm

USA

Alexander Fletcher

UK

\section{Alexander Wolff}

Germany

Alexandre Bureau

Canada

Ali Anaissi

Australia

Sharma Alok

Australia

Stephen Altschul

USA

Alun Thomas

USA

Amarda Shehu

USA

Correspondence: dirk.krueger@biomedcentral.com

BioMed Central, Floor 6, 236 Gray's Inn Road, London WC1X 8HB, UK 
Fernando Amat

USA

Anthony Mathelier

Canada

Amjad Ali

Pakistan

Ana Pavel

USA

Ananda Roy

USA

Ananth Kalyanaraman

USA

Anders Berglund

USA

Andigoni Malousi

Greece

András Aszódi

Austria

Andras Fiser

USA

Andrea Rau

France

Andreas Heger

UK

Andreas Keller

Germany

Andreas Prlic

USA

Andrei Lupas

Germany

E. Andres Houseman

USA

Andrew Dowsey

UK

Andrew Jaffe

USA

Andrew Allen

USA

Andrew Thomas

Brazil

Anoop Mayampurath

USA

Anna Goldenberg

Canada
Anna Ritz

USA

Anne Biton

USA

Anton Camacho

UK

Antonio Jose Jimeno Yepes

Australia

Ao Li

China

Alexandre Francisco

Portugal

Andrey Ptitsyn

Qatar

Alejandro Nato

USA

Lucia Chemes

Argentina

Ari Loytynoja

Finland

Arkadiusz Gertych

USA

Arndt Von Haeseler

Austria

Arne Elofsson

Dominica

Lars Arvestad

Sweden

Andreas Schlueter

Germany

Alexander Sczyrba

Germany

Mohammad Khan

Malaysia

Sourav S Bhowmick

Singapore

Alan Talevi

Argentina

Georgios Athanasiadis

Denmark

Hannah Nicholas

Australia

Merridee Wouters

Australia
Lachlan Coin

Australia

Scott Ritchie

Australia

Shu-Kay Angus Ng

Australia

Vanessa Teague

Australia

Matthew Ritchie

Australia

Xiaohui Tao

Australia

Andrew Collins

Australia

Louis Sherman

Australia

David Duffy

Australia

David Balding

Australia

Konsta Duesing

Australia

Ritaban Dutta

Australia

Yadav Sapkota

Australia

Gábor Mészáros

Austria

Florian Pauler

Austria

Rainer Schuhmacher

Austria

Gregory Ewing

Austria

Christian Partl

Austria

Avinash K Shanmugam

USA

Benjamin Chain

UK

Benjamin Linard

UK

Bas Van Breukelen

Netherlands 
Rolf Backofen

Germany

Bairong Shen

China

Sandro Banfi

Italy

Zhirong Bao

USA

Basir Shariat

USA

Daniel Baum

Germany

Babajan Banaganapalli

Saudi Arabia

Robert Beiko

Canada

Jan De Neve

Belgium

Yvan Saeys

Belgium

Dieter Deforce

Belgium

Karoline Faust

Belgium

Gipsi Lima Mendez

Belgium

Benedicte Elena-Herrmann

France

Benoit Ballester

France

Nir Ben Tal

Israel

Bernd Fischer

Germany

Denis Bertrand

Singapore

Bharath Srinivasan

USA

Binghuang Cai

USA

Velumani Bhuvaneswari

India

Ali Bilgin

USA
Binay Panda

India

Bingqiang Liu

Canada

Bingshan Li

USA

Vijay Garg

India

Monika Michalovova

USA

Bernard Pope

Australia

Burkhard Morgenstern

Germany

Ornulf Borgan

Norway

Anne-Laure Boulesteix

Germany

Babak Alipanahi

Canada

Bo Peng

USA

Yu Xia

Canada

Douglas Pires

Brazil

Hernandes Carvalho

Brazil

Benilton Carvalho

Brazil

Raydonal Ospina

Brazil

Daniel Oliveira

Brazil

Brett Lidbury

Australia

Brian Connolly

USA

Fiona Brinkman

Canada

Bruno Nevado

UK

Brian Tjaden

USA
Veselka Boeva

Bulgaria

Donald H Burke

USA

Carsten Kemena

Germany

Can Kesmir

Netherlands

Chanchal Mitra

India

Chunyu Jin

USA

Bhagwati Gupta

Canada

Earl Brown

Canada

Christian Frech

Canada

Herbert $\mathbf{H}$ Tsang

Canada

Dionne Aleman

Canada

Marek Laskowski

Canada

Jennifer Mitchell

Canada

Hagit Shatkay

Canada

Shaun Jackman

Canada

Alan Moses

Canada

Donovan Parks

Canada

Xuhua Xia

Canada

Carl Kingsford

USA

Carlo Manzo

Spain

Carlo Baldassi

Italy

Carolina Wahlby

USA 


\begin{tabular}{|c|c|c|}
\hline $\begin{array}{l}\text { Rita Casadio } \\
\text { Italy }\end{array}$ & $\begin{array}{l}\text { Ming Chen } \\
\text { China }\end{array}$ & $\begin{array}{l}\text { Simon Rayner } \\
\text { China }\end{array}$ \\
\hline $\begin{array}{l}\text { Chris Benner } \\
\text { USA }\end{array}$ & $\begin{array}{l}\text { Huaqiang Du } \\
\text { China }\end{array}$ & $\begin{array}{l}\text { Wei Jiang } \\
\text { China }\end{array}$ \\
\hline $\begin{array}{l}\text { Christian Blouin } \\
\text { Canada }\end{array}$ & $\begin{array}{l}\text { Zhi Lu } \\
\text { China }\end{array}$ & $\begin{array}{l}\text { Fei Liu } \\
\text { China }\end{array}$ \\
\hline $\begin{array}{l}\text { Carlos Castro } \\
\text { Spain }\end{array}$ & $\begin{array}{l}\text { Yc Wang } \\
\text { China }\end{array}$ & $\begin{array}{l}\text { Ching Wan Lam } \\
\text { Hong Kong }\end{array}$ \\
\hline $\begin{array}{l}\text { Celia Greenwood } \\
\text { Canada }\end{array}$ & $\begin{array}{l}\text { Xuan Xiao } \\
\text { China }\end{array}$ & $\begin{array}{l}\text { Chloé-Agathe Azencott } \\
\text { France }\end{array}$ \\
\hline $\begin{array}{l}\text { Ching-Fen Jiang } \\
\text { Taiwan }\end{array}$ & $\begin{array}{l}\text { Zu-Guo Yu } \\
\text { China }\end{array}$ & $\begin{array}{l}\text { Christian Paroissin } \\
\text { France }\end{array}$ \\
\hline $\begin{array}{l}\text { Brad Chapman } \\
\text { USA }\end{array}$ & $\begin{array}{l}\text { Min Li } \\
\text { China }\end{array}$ & $\begin{array}{l}\text { Christian Remmele } \\
\text { Germany }\end{array}$ \\
\hline $\begin{array}{l}\text { Charlotte Soneson } \\
\text { Switzerland }\end{array}$ & $\begin{array}{l}\text { Yaning Yang } \\
\text { China }\end{array}$ & $\begin{array}{l}\text { Christian Schlötterer } \\
\text { Austria }\end{array}$ \\
\hline $\begin{array}{l}\text { Chava Kimchi-Sarfaty } \\
\text { USA }\end{array}$ & $\begin{array}{l}\text { Wei Chen } \\
\text { China }\end{array}$ & $\begin{array}{l}\text { Christine Nardini } \\
\text { Italy }\end{array}$ \\
\hline $\begin{array}{l}\text { Jun Chen } \\
\text { USA }\end{array}$ & $\begin{array}{l}\text { Quan Zou } \\
\text { China }\end{array}$ & $\begin{array}{l}\text { Christine Staiger } \\
\text { Netherlands }\end{array}$ \\
\hline $\begin{array}{l}\text { Chen Kaesar } \\
\text { Israel }\end{array}$ & $\begin{array}{l}\text { Yan Fu } \\
\text { China }\end{array}$ & $\begin{array}{l}\text { Ambroise Christophe } \\
\text { France }\end{array}$ \\
\hline $\begin{array}{l}\text { Cheng Chen } \\
\text { USA }\end{array}$ & $\begin{array}{l}\text { Xuehai Hu } \\
\text { China }\end{array}$ & $\begin{array}{l}\text { Christophe Beroud } \\
\text { France }\end{array}$ \\
\hline $\begin{array}{l}\text { Jianlin Cheng } \\
\text { USA }\end{array}$ & $\begin{array}{l}\text { Yihui Luan } \\
\text { China }\end{array}$ & $\begin{array}{l}\text { Christopher Funk } \\
\text { USA }\end{array}$ \\
\hline $\begin{array}{l}\text { Yizong Cheng } \\
\text { USA }\end{array}$ & $\begin{array}{l}\text { Lei Qu } \\
\text { China }\end{array}$ & $\begin{array}{l}\text { Chuck Cannon } \\
\text { USA }\end{array}$ \\
\hline $\begin{array}{l}\text { Chiara Romualdi } \\
\text { Italy }\end{array}$ & $\begin{array}{l}\text { Hong-Bin Shen } \\
\text { China }\end{array}$ & $\begin{array}{l}\text { James Cimino } \\
\text { USA }\end{array}$ \\
\hline $\begin{array}{l}\text { Chih-Hsuan Wei } \\
\text { USA }\end{array}$ & $\begin{array}{l}\text { Yi Liu } \\
\text { China }\end{array}$ & $\begin{array}{l}\text { Cinzia Pizzi } \\
\text { Italy }\end{array}$ \\
\hline $\begin{array}{l}\text { Chih Lee } \\
\text { USA }\end{array}$ & $\begin{array}{l}\text { Shaowu Zhang } \\
\text { China }\end{array}$ & $\begin{array}{l}\text { Cinzia Viroli } \\
\text { Italy }\end{array}$ \\
\hline $\begin{array}{l}\text { Rayan Chikhi } \\
\text { France }\end{array}$ & $\begin{array}{l}\text { Zhiwei Cao } \\
\text { China }\end{array}$ & $\begin{array}{l}\text { Charles Kervrann } \\
\text { France }\end{array}$ \\
\hline $\begin{array}{l}\text { Jin Gu } \\
\text { China }\end{array}$ & $\begin{array}{l}\text { Rui Jiang } \\
\text { China }\end{array}$ & $\begin{array}{l}\text { Caleb Stein } \\
\text { USA }\end{array}$ \\
\hline $\begin{array}{l}\text { Zhi-Ping Liu } \\
\text { China }\end{array}$ & $\begin{array}{l}\text { Yanghua Xiao } \\
\text { China }\end{array}$ & $\begin{array}{l}\text { Claudio Donati } \\
\text { Italy }\end{array}$ \\
\hline $\begin{array}{l}\text { Yinglei Song } \\
\text { China }\end{array}$ & $\begin{array}{l}\text { Jianhua Xu } \\
\text { China }\end{array}$ & $\begin{array}{l}\text { Christina Leslie } \\
\text { USA }\end{array}$ \\
\hline $\begin{array}{l}\text { Xingming Zhao } \\
\text { China }\end{array}$ & $\begin{array}{l}\text { Yu Xue } \\
\text { China }\end{array}$ & $\begin{array}{l}\text { Che-Lun Hung } \\
\text { Taiwan }\end{array}$ \\
\hline
\end{tabular}


Cristina Marino-Buslje

Argentina

Carlos Silla

Brazil

Hanbo Chen

USA

Colin Clarke

Ireland

Colin Smith

Germany

Matteo Comin

Italy

Oliver Philipp

Germany

Cheolwoo Park

USA

Cedric Saule

Germany

Casey Greene

USA

C. Titus Brown

USA

Ching-Ti Liu

USA

Qing-Hua Cui

China

Fabio Cunial

Germany

Chris Upton

Canada

Ching-Wei Wang

Taiwan

Cyril Grouin

France

Chenggang Yu

USA

Jan Brezovsky

Czech Republic

Jiri Klema

Czech Republic

Diana Hendrickx

Netherlands

Donovan Parks

Australia
Thomas Dandekar

Germany

Daniel Aguilar

USA

Daniel Sage

Switzerland

Daniel Falush

UK

David Price

USA

David Ardell

USA

David Blair

USA

David La

USA

David Hall

USA

David Meckes

USA

David A Eberhard

USA

Davide Risso

USA

Danny Barash

Israel

Dukka Kc

USA

Debasisa Mohanty

India

Deepak Ayyala

USA

Deepak Singla

USA

Denis Bauer

Australia

Huangdi Yi

China

Jonas Andreas Sibbesen

Denmark

Dennis Hazelett

USA

Dennis Welker

USA
Des Higgins

Ireland

Deyvid Amgarten

Brazil

Domenico L Gatti

USA

Damyanthi Herath

Australia

Dragos Horvath

France

Diana Bahia

Brazil

Diana Sima

Belgium

Dick De Ridder

Netherlands

Susan Ghiassian

USA

Dinesh Kumar

USA

Dirk Valkenborg

Belgium

Daofeng Li

USA

Daniel Machado

Portugal

Dominik Heider

Germany

Dominik Schrempf

Austria

Nadezhda Doncheva

Germany

Dong Si

USA

Dong Wang

USA

Deguang Kong

USA

Subramanian Krishnakumar

India

Dean Rowe-Magnus

USA

Dario Strbenac

Australia 
Deqiang Sun
USA

Duccio Fanelli

Italy

Murat Dundar

USA

Micah Dunthorn

Germany

Dannie Durand

USA

Derrick Wood

USA

Di Wu

USA

Ingo Ebersberger

Germany

Edyta Koscianska

Poland

Eldon Emberly

Canada

Can Yang

USA

Ralf Eggeling

Germany

Etienne Kornobis

Germany

Eleni Giannoulatou

UK

Eduardo Andrés-León

Spain

Emily Prud'Hommeaux

USA

Emily Chia-Yu Su

Taiwan

Ellis Patrick

USA

Erica Marie Hartmann

USA

Erich Baker

USA

Eric Wait

USA

Erliang Zeng

USA
Esmaeil Ebrahimie

Australia

Eric Vallender

USA

Eytan Domany

Israel

Fuyong Xing

USA

Fabian Sievers

Ireland

Falk Hildebrand

Germany

Falk Schreiber

Australia

Ruzong Fan

USA

Mario Fares

Ireland

Alexander Favorov

Russia

Francesca Cordero

Italy

Francisco Couto

Portugal

Feifan Liu

USA

Francisco Enguita

Portugal

Ferhat Ay

USA

Filippo Utro

Switzerland

Pekka Marttinen

Finland

Petri Toronen

Finland

Fiona McCarthy

USA

Federico Giorgi

USA

Folker Meyer

USA

Dimitrios Fotiadis

Greece
Francesco Pappalardo Italy

Guy Perriere

France

Yann Ponty

France

Eva Dhondt

France

Julien Gobeill

France

Benjamin Roche

France

Guillaume Achaz

France

Arthur Tenenhaus

France

Daniel Jost

France

Pierre Peterlongo

France

Alexandre De Brevern

France

Bruno Toupance

France

Thomas Boudier

France

Laurent Brehelin

France

Mathieu Gautier

France

Claudine Medigue

France

Caroline Berard

France

Francesca Martella Italy

Tzong-Yi Lee

Taiwan

Francisco De La Vega

USA

Franck Picard

France

Fred Hamprecht

Germany 
Saskia Freytag

Australia

Holger Fröhlich

Germany

Feixiong Cheng

USA

Gibran Hemani

UK

Gabriel Cristóbal

Spain

Gabriel Renaud

Germany

Julien Gagneur

Germany

Ganesh Sahoo

India

Vincent Gardeux

Switzerland

Gerard Cagney

Ireland

Michaela Bayerlova

Germany

Ulrich Stelzl

Germany

Manuel Holtgrewe

Germany

André Scherag

Germany

Jochen Blom

Germany

Jaime Huerta-Cepas

Germany

Bryan Downie

Germany

Marcel Martin

Germany

Harald Binder

Germany

Wolfgang Förstner

Germany

Stefan Paulus

Germany

Axel Benner

Germany
Julien Dutheil

Germany

Friedrich Foerster

Germany

Frank Kramer

Germany

Astrid Wachter

Germany

Marcus Dittrich

Germany

Marcus Lechner

Germany

Mariana Neves

Germany

Ezgi Karaca

Germany

Benedikt Brors

Germany

Matthias Ganzinger

Germany

Jerzy Dyczkowski

Germany

Kaustubh Patil

Germany

Ursula Kummer

Germany

Johannes Köster

Germany

Michael Seifert

Germany

Graham Hatfull

Brazil

Greg Finak

USA

Giorgio Bertorelle

Italy

Ghislain Bidaut

France

Gianfranco Politano

Italy

Georgi Marinov

USA

George Michailidis

USA
Gokmen Zararsiz

Turkey

Mehmet Gonen

USA

Gonzalo Ruz

Chile

Georgios Tsaousis

Greece

Margarita Theodoropoulou Greece

Gordon Robertson

Canada

Michael Gromiha

India

Gustavo Rosania

USA

Thierry Zozio

France

Guillemette Marot

France

Gur Yaari

Israel

Gyorgy Abrusan

Hungary

Yuanfang Guan

USA

Holly Bik

UK

Henry Wood

UK

Hien Nguyen

Australia

Hae Uh

Netherlands

Ran Libeskind-Hadas

USA

Chen Haifeng

China

Hailin Chen

USA

Haiquan Li

USA

Hajk-Georg Drost

Denmark 


\author{
Thierry Hamon \\ France \\ Hanchuan Peng \\ USA
}

Hane Lee

USA

Jikai Lei

USA

Hannah Christensen

UK

Hans Kestler

Germany

Hao Zheng

USA

M Hasinur Rahaman Khan

Bangladesh

Haixu Tang

USA

Bernhard Haubold

Germany

Hao Chen

Hong Kong

Huaichun Wang

Canada

Heba Saadeh

UK

Heewook Lee

USA

Heike Siebert

Germany

Heiner Klingenberg

Germany

Heng Wang

USA

Heping Zhang

USA

Hesam Montazeri

Switzerland

Itaru Hirai

Japan

Hyun Jung Park

USA

Hiqmet Kamberaj Macedonia

The Former Yugoslav Republic Of
Hailong Zhu

China

Hidetoshi Matsui

Japan

Hongmin Cai

China

Haimao Zhan

USA

Xiaowen Chu

Hong Kong

Xiaodan Fan

Hong Kong

Hongyu Zhao

USA

Roman Hornung

Germany

Lin Hou

USA

Houtan Noushmehr

Brazil

Hai Su

USA

Xia $\mathrm{Hu}$

USA

Hu Qian-Nan

China

Hui Huang

USA

Yuan Huang

USA

Hunter Moseley

USA

Hongyan Xu

USA

Hamid Younesy

Canada

Henry Zhang

USA

Hongyi Zhou

USA

Iain Manfield

UK

Ihor Smal

Netherlands
Thomas Simpson

UK

Iddo Friedberg

USA

Ivan Dotu

USA

Ilya Serebriiskii

USA

Ilan Samish

Isreal

Ioannis Iliopoulos

Greece

Imhoi Koo

USA

Ina Koch

Germany

Narayanaswamy Srinivasan India

Hamsa Priya Mohana Sundaram India

Gajendra Raghava

India

Achuthsankar S Nair

India

Rameshkumar Krishnamoorthy India

B. Jayaram

India

Balraj Mittal

India

Inke König

Germany

Inken Wohlers

Germany

Ion Mandoiu

USA

Cathal Seoighe

Ireland

Dietrich Rebholz-Schuhmann

Ireland

Irene Tischer

Colombia

Isaam Saeed

Australia 
Tal Pupko

Israel

Firas Swidan

Israel

Sol Efroni

Israel

Eyal Privman

Israel

Tamir Tuller

Israel

Giorgio Valentini

Italy

Luisa Di Paola

Italy

Flavia Guzzo

Italy

Silvio Tosatto

Italy

Cristian Del Fabbro

Italy

Piero Fariselli

Italy

Pier Luigi Martelli

Italy

Pietro Di Lena

Italy

Andrea Calabria

Italy

Pasquapina Ciarmela

Italy

Serafina Massari

Italy

Kimihito Ito

Japan

Ivan Kulakovskiy

Russia

Ivan Limongelli

Italy

Ivan Merelli

Italy

Jörg Ackermann

Germany

Jiyuan An

USA
Pjotr Prins

Netherlands

Jorge Duitama

Colombia

Yu-Chieh Liao

Taiwan

Nivedita Deo

USA

James Costello

USA

James Lara

USA

Jian Zhang

USA

Jamie Sherman

Canada

Jan Fostier

Belgium

Jan Hasenauer

Germany

Naoko Takezaki

Japan

Fumihiko Takeuchi

Japan

Masanori Arita

Japan

Wataru Nemoto

Japan

Yoshimasa Tsuruoka

Japan

Nozomi Nagano

Japan

Timo Lassmann

Japan

Jun Zhang

Japan

Michiaki Hamada

Japan

Yang Yang

Japan

Tadashi Ando

Japan

Satya Arjunan

Japan
Jared Simpson

Canada

Jaroslaw Zola

USA

Javed Agrewala

India

Jayajit Das

USA

Jared Burks

USA

João André Carriço

Portugal

Jair Cervantes

Mexico

Jason Kahn

USA

Jean Yang

Australia

Jean-Christophe Lambry

France

Jean-Jacques Daudin

France

Robert Jernigan

USA

Jérôme Ambroise

Belgium

Jianfeng Pei

China

Jianjiong Gao

USA

John Hartman

USA

Jiangning Song

Australia

Yu Jiang

USA

Jiehuan Sun

USA

Jieyue Li

USA

Jinbo Xu

USA

Jin Chu Wu

USA 
Jing Tang

Finland

Jing-Hao Xue

UK

Victor Jin

USA

Jin Yu Li

USA

Jonas Korlach

USA

Jie Liang

USA

John-Marc Chandonia

USA

Jarek Meller

USA

John Murray

USA

Juanma Vaquerizas

Germany

Joan Maynou

Spain

Joel Tellinghuisen

USA

Joeri Ruyssinck

Belgium

Johannes Droege

Germany

Johannes Waage

Denmark

Jonathan Keith

USA

Jos Boekhorst

Netherlands

Joshua Payne

Switzerland

Jean Peccoud

USA

Jianhua Ruan

USA

Junhee Seok

South Korea

Jiansen Jiang

USA
José Patané

Brazil

Florian Jug

Germany

Juilee Thakar

USA

Julia Perera Bel

Germany

Juliana Bernardes

France

Julio Banga

USA

Suckjoon Jun

USA

Justin Zook

USA

James Taylor

USA

Jianxin Wang

China

Jingyuan Song

China

Janos Zempleni

USA

Kim-Anh Lê Cao

Australia

Kristina Hettne

Netherlands

Koji Kadota

Japan

Karin Verspoor

Australia

Karina S. Machado

Brazil

Karsten Hiller

Luxembourg

Katerina Kechris

USA

Kazutaka Katoh

Japan

Kent Riemondy

USA

Karen Ross

USA
Kevin Yip

Hong Kong

Glass $\mathrm{K}$

USA

Kin Fai Au

USA

Kirk Roberts

USA

Kishore Mosaliganti

USA

Kevin Keegan

USA

Pavel Skums

USA

Klaus Jung

Germany

Marius Kloft

Germany

Kailin Tang

China

Karina Mayorga

Mexico

Tamas Korcsmaros

Hungary

Sukjoon Yoon

South Korea

Sun Shim Choi

South Korea

Buhm Han

South Korea

Jaehee Kim

South Korea

Wonkuk Kim

South Korea

Sergey Koren

USA

Kostas Tsirigos

Sweden

Khalid Raza

India

Srikrishna Subramanian India

Kristin Ayers

UK 
Kristof Engelen

Belgium

Sergey Ovchinnikov

USA

Kristoffer Sahlin

Sweden

Konstantinos Spiliopoulos

USA

Kalliopi Tsafou

USA

Kentaro Tomii

Japan

Stefan Kurtz

Germany

Anthony Kusalik

Canada

Kai Wang

USA

Ken Witwer

USA

Yung-Keun Kwon

South Africa

Kristine Wylie

USA

Kazunori Yamada

Japan

Lan Du

Australia

Lars-Gustav Snipen

Norway

Lars Bongo

Norway

Jessica Larson

USA

Laura Saba

USA

Laurent Jacob

France

Mario Lauria

Italy

Layla Oesper

USA

Leandro Guerrero

South Africa
Robert Hoehndorf

UK

Lee Kamentsky

USA

Seunggeun Lee

USA

Lei Song

USA

Leighton Pritchard

UK

Matej Lexa

Czech Republic

Luís F De Figueiredo

UK

Lana Garmire

USA

Li Chen

USA

Li Beißbarth

Germany

Li Wang

USA

Xing Li

USA

Chun Liang

USA

Guangdi Li

China

Lili Wang

Canada

Lin Xu

USA

Xiang Li

USA

Yushi Liu

USA

Yazhi Liu

China

Liyang Diao

USA

Ying Li

China

Kenli Li

China
Long Qu

USA

Loredana Martignetti

France

Lorenzo Tattini

Italy

Luca Pinello

USA

Lynn Schriml

USA

Li Song

USA

Liang $\mathrm{Hu} \mathrm{Qu}$

China

Lu Huang

USA

Lucas Carey

Spain

Luca Toldo

Germany

Luciano Cascione

Switzerland

Luis Sanchez-Pulido

UK

Luke Czapla

USA

Lun-Ching Chang

USA

Jiawei Luo

China

Lei Xie

USA

Lynn Lin

USA

Minh Duc Cao

Australia

Martijn Huynen

Netherlands

Marek Kowal

Poland

Miron Kursa

Poland

Markus Meringer

Germany 
Mark Wass

UK

Mark Ragan

Australia

Mar Rodriguez Girondo

USA

Mathieu Rouard

Italy

Monica Santamaria

Italy

Marc Santolini

USA

Marco Scutari

UK

Nadia El-Mabrouk

Canada

Kathy Macropol

USA

Mahreen Arooj

Australia

Wolfgang Gerlach

USA

Majid Kazemian

USA

George Makhatadze

USA

Manabu Torii

USA

Manish Kumar

India

Giovanni Manzini

Italy

Marcella Attimonelli

Italy

Blanca Caminero

Spain

Marie-Paule Lefranc

France

Mark D. Robinson

Switzerland

Mark Van De Wiel

Netherlands

Martin Hofmann-Apitius

Germany
Martin Maska

Czech Republic

Martin Weigt

France

Joaquim Martins Jr

Brazil

Martin Mascher

Germany

Masaaki Kotera

Japan

Matteo Benelli

Italy

Matteo Figliuzzi

France

Matthew Bennett

USA

Matthew Macmanes

USA

Matthew McCall

USA

Matthias Wolf

Germany

Louxin Zhang

Singapore

Maxim Barenboim

Germany

Johanna Mazur

Germany

Manoj Bhasin

USA

Min Chen

USA

Maria Chikina

USA

Paul McMurdie

USA

Matthias Döring

Germany

Mark Ebbert

USA

Megan Orr

USA

Meik Kunz

Germany
Menachem Fromer

USA

Mengjie Chen

USA

Enrique Hernandez-Lemus

Mexico

Rafael Villalobos

Mexico

Michelle Giglio

USA

Mohammad Ganjtabesh

Iran

Melissa Gymrek

USA

Michael Herman

USA

Micha Bayer

UK

Michael Götze

Germany

Michael Hoopmann

USA

Michal Okoniewski

Switzerland

Michal Brylinski

USA

Michelangelo Ceci

Italy

Mikael Salson

France

Mikhail Spivakov

UK

Mile Sikic

Croatia

Ming $\mathrm{Hu}$

USA

Minh Nguyen

Singapore

Ramanathan Sowdhamini

India

Mira Han

USA

Julie Mitchell

USA 
Matloob Khushi

Australia

Mahbubul Majumder

USA

Ram Podicheti

USA

Mohamed Elati

France

Monica Chagoyen

Spain

Morihiro Hayashida

Japan

Marco Punta

UK

Reaz Uddin

Pakistan

Madis Rumming

Germany

Moisés Santillan

Mexico

Marcel H. Schulz

Germany

Margaret Taub

USA

Mu Gao

USA

T. Murali

USA

Mark Winter

USA

Maria-Eirini Pandelia

USA

Nadim Ajami

USA

Nima Aghaeepour

USA

Ali Najafi

Iran

Nam Nguyen

USA

Nan Zhao

USA

Nanjiang Shu

Sweden
Natali Gulbahce

USA

Nathalie Villa-Vialaneix

France

Nathaniel Snyder

USA

Saket Navlakha

USA

Neo Christopher Chung

USA

Neil Mac Parthaláin

UK

Andrew Wong

USA

Nestoras Karathanasis

Greece

Gert Vriend

Netherlands

Peter Horvatovich

Netherlands

K. Anton Feenstra

Netherlands

Casper Albers

Netherlands

Hinda Haned

Netherlands

David Welch New

Zealand

Nicola Segata

Italy

Nicolas Heck

France

Ning Chen

China

Nianjun Teng

China

Ning Leng

USA

Nan Li

USA

Nan Lin

USA

Daniel Noguera

USA
Finn Drabløs

Norway

Nico Pfeifer

Germany

Nima Pouladi

USA

Nurcan Tuncbag

USA

Grégory Nuel

France

Ruth Nussinov

USA

Osamu Gotoh

Japan

Olga Vitek

USA

Catalina Tudor

USA

Jonathon O'Brien

USA

David Ochoa

UK

Michael Ochs

USA

Olivier Cinquin

USA

Olivier Gevaert

USA

Kazuma Okada

Japan

Olaf Bininda-Emonds

Germany

Oliver Schilling

Germany

Omer An

UK

Brian Ondov

USA

Ong Mei-Lyn

Singapore

Oswaldo Trelles

Spain

Piotr Makowski

Poland 
Pablo Minguez

Germany

Paul Aiyetan

USA

Pal Sætrom

Norway

Palok Aich

India

Bogdan Pasaniuc

USA

Andrea Passerini

Italy

Patricio Oyarzun

Chile

Patthy Laszlo

Hungary

Paul Boutros

Canada

Paula Veríssimo

Portugal

Florencio Pazos

Spain

Patrick Chain

USA

Piotr Cieplak

USA

Marcin Skwark

Finland

Shyamal Peddada

USA

Pedro Ballester

France

Peng Qiu

USA

Peter Bajcsy

USA

Peter White

USA

Petra Knaup

Germany

Phil Lee

USA

Philippe Gambette

France
Barry Smith

USA

Pietro Franceschi

Italy

Pingzhao Hu

USA

Pingzhao Hu

Canada

Peng Jiang

USA

Paulo Pierry

Brazil

Ilona Wandzik

Poland

Henryk Rybinski

Poland

Sandor Pongor

Italy

Matthias Erwin Futschik

Portugal

Pratyaksha Wirapati

Switzerland

Predrag Radivojac

USA

Pedro Ribeiro

Portugal

Patrick Schloss

USA

P. Shanmugavadivu

India

Pierre Zweigenbaum

France

Ping Zhang

USA

Qian Wang

USA

Qibin Zhang

USA

Michele Ceccarelli

Qatar

Qiangjun Zhou

USA

Ji Qi

China
Qing Zhao

USA

Jing Qin

Hong Kong

Li-Xuan Qin

USA

Qiongshi Lu

USA

Jing Qiu

USA

Xin Qi

USA

Yifei Qi

USA

Quan Chen

USA

Quang Le

UK

Ranjit Bahadur India

Roman Koning

Netherlands

Robert Nowak

Poland

Ralf Schmid

UK

Rachel Schwartz

USA

Rainer Merkl

Germany

Rainer Spang

Germany

Rakesh Joshi

India

Ramakrishnan C, India

Ramon Diaz-Uriarte

Spain

Franck Rapaport

USA

Tobias Rausch

Germany

Ravi Shankar India 


\begin{tabular}{|c|c|c|}
\hline $\begin{array}{l}\text { Robert Rebhun } \\
\text { USA }\end{array}$ & $\begin{array}{l}\text { Raman Parkesh } \\
\text { India }\end{array}$ & $\begin{array}{l}\text { Alejandro Schaffer } \\
\text { USA }\end{array}$ \\
\hline $\begin{array}{l}\text { Ren-Hua Chung } \\
\text { Taiwan }\end{array}$ & $\begin{array}{l}\text { Raul Tonda } \\
\text { Spain }\end{array}$ & $\begin{array}{l}\text { Alexander Schliep } \\
\text { USA }\end{array}$ \\
\hline $\begin{array}{l}\text { Rebecca Hunt } \\
\text { Germany }\end{array}$ & $\begin{array}{l}\text { Rui Zhong } \\
\text { USA }\end{array}$ & $\begin{array}{l}\text { Scott Doyle } \\
\text { USA }\end{array}$ \\
\hline $\begin{array}{l}\text { Reinhard Guthke } \\
\text { Germany }\end{array}$ & $\begin{array}{l}\text { Ruslan Kalendar } \\
\text { Finland }\end{array}$ & $\begin{array}{l}\text { Sebastian Kaiser } \\
\text { USA }\end{array}$ \\
\hline $\begin{array}{l}\text { Remo Sanges } \\
\text { Italy }\end{array}$ & $\begin{array}{l}\text { Ekaterina Chernyaeva } \\
\text { Russia }\end{array}$ & $\begin{array}{l}\text { Sebastian Maurer-Stroh } \\
\text { Singapore }\end{array}$ \\
\hline $\begin{array}{l}\text { Rudy Guerra } \\
\text { USA }\end{array}$ & $\begin{array}{l}\text { Rohit Bhargava } \\
\text { USA }\end{array}$ & $\begin{array}{l}\text { Selvaraj Samuel } \\
\text { India }\end{array}$ \\
\hline $\begin{array}{l}\text { Riccardo Bellazzi } \\
\text { Italy }\end{array}$ & $\begin{array}{l}\text { Rong Xu } \\
\text { USA }\end{array}$ & $\begin{array}{l}\text { Jose Seoane } \\
\text { USA }\end{array}$ \\
\hline $\begin{array}{l}\text { Richard Boys } \\
\text { UK }\end{array}$ & $\begin{array}{l}\text { Shu-Kay Ng } \\
\text { Australia }\end{array}$ & $\begin{array}{l}\text { Miljana Tanic } \\
\text { Serbia }\end{array}$ \\
\hline $\begin{array}{l}\text { Richard White } \\
\text { USA }\end{array}$ & $\begin{array}{l}\text { Sebastian Schmeier } \\
\text { New Zealand }\end{array}$ & $\begin{array}{l}\text { Sergio Marco Garrido } \\
\text { France }\end{array}$ \\
\hline $\begin{array}{l}\text { Rongjian Li } \\
\text { USA }\end{array}$ & $\begin{array}{l}\text { Shailesh Tripathi } \\
\text { UK }\end{array}$ & $\begin{array}{l}\text { Andrew Severin } \\
\text { USA }\end{array}$ \\
\hline $\begin{array}{l}\text { Robert Frost } \\
\text { USA }\end{array}$ & $\begin{array}{l}\text { Sabarinathan Radhakrishnan } \\
\text { Denmark }\end{array}$ & $\begin{array}{l}\text { Shubhra Ghosh Dastidar } \\
\text { India }\end{array}$ \\
\hline $\begin{array}{l}\text { Rob Patro } \\
\text { USA }\end{array}$ & $\begin{array}{l}\text { Mohd Saberi Mohamad } \\
\text { Malaysia }\end{array}$ & $\begin{array}{l}\text { Sarah Goodwin } \\
\text { USA }\end{array}$ \\
\hline $\begin{array}{l}\text { Robert Kofler } \\
\text { Austria }\end{array}$ & $\begin{array}{l}\text { Saeed Salem } \\
\text { USA }\end{array}$ & $\begin{array}{l}\text { Yuanpu Xie } \\
\text { USA }\end{array}$ \\
\hline $\begin{array}{l}\text { Robert Leaman } \\
\text { USA }\end{array}$ & $\begin{array}{l}\text { Salvador Capella-Gutíerrez } \\
\text { Spain }\end{array}$ & $\begin{array}{l}\text { Yuk Sham } \\
\text { USA }\end{array}$ \\
\hline $\begin{array}{l}\text { Robert Edgar } \\
\text { USA }\end{array}$ & $\begin{array}{l}\text { David Sankoff } \\
\text { Canada }\end{array}$ & $\begin{array}{l}\text { Shandar Ahmad } \\
\text { Japan }\end{array}$ \\
\hline $\begin{array}{l}\text { Robert Cooper } \\
\text { USA }\end{array}$ & $\begin{array}{l}\text { Santi Garcia-Vallvé } \\
\text { Spain }\end{array}$ & $\begin{array}{l}\text { Steffen Heber } \\
\text { USA }\end{array}$ \\
\hline $\begin{array}{l}\text { Rodolfo Negri } \\
\text { Italy }\end{array}$ & $\begin{array}{l}\text { Sarah Cohen Boulakia } \\
\text { France }\end{array}$ & $\begin{array}{l}\text { Shishi Luo } \\
\text { USA }\end{array}$ \\
\hline $\begin{array}{l}\text { Rodrigo Guarischi-Sousa } \\
\text { Brazil }\end{array}$ & $\begin{array}{l}\text { Sarah Hird } \\
\text { USA }\end{array}$ & $\begin{array}{l}\text { Shishir Gupta } \\
\text { Germany }\end{array}$ \\
\hline $\begin{array}{l}\text { Rohit Vashisht } \\
\text { USA }\end{array}$ & $\begin{array}{l}\text { Sara Mostafavi } \\
\text { Canada }\end{array}$ & $\begin{array}{l}\text { Xingjie Shi } \\
\text { China }\end{array}$ \\
\hline $\begin{array}{l}\text { Roland Krause } \\
\text { Luxembourg }\end{array}$ & $\begin{array}{l}\text { Sarwar Kamal } \\
\text { Bangladesh }\end{array}$ & $\begin{array}{l}\text { Sheng Li } \\
\text { USA }\end{array}$ \\
\hline $\begin{array}{l}\text { Ron Wehrens } \\
\text { Netherlands }\end{array}$ & $\begin{array}{l}\text { Vladimir Bajic } \\
\text { Saudi Arabia }\end{array}$ & $\begin{array}{l}\text { Shuai Huang } \\
\text { USA }\end{array}$ \\
\hline $\begin{array}{l}\text { Rachid Ounit } \\
\text { USA }\end{array}$ & $\begin{array}{l}\text { Sam Scarpino } \\
\text { USA }\end{array}$ & $\begin{array}{l}\text { Shuangge Ma } \\
\text { USA }\end{array}$ \\
\hline
\end{tabular}


Siamak Tafavogh

USA

Silke Sperling

Germany

Simon Andrews

Simona E. Rombo

Italy

Simona Rombo

Italy

Andreas Wilm

Singapore

Weimiao Yu

Singapore

Min Wu

Singapore

Janos Kriston-Vizi

Singapore

Lit-Hsin Loo

Singapore

Erik Cambria

Singapore

Jung-Jae Kim

Singapore

Shailza Singh

India

Samad Jahandideh

USA

Sidharth Chopra

India

Sara Madeira

Portugal

Simone Marsili

Spain

Sumit Middha

USA

Stefano Monti

USA

Gordon Smyth

Australia

Sorin Draghici

USA

Johannes Soeding

Germany
Solon Pissis

UK

Song Li

USA

Salvador Capella-Gutiérrez

Spain

Ivan Junier

Spain

Samira Jaeger

Spain

Juan Jesus Perez

Spain

Jana Selent

Spain

Marc A. Marti-Renom

Spain

David Juan

Spain

Pilar Gonzalez-Gomez

Spain

María Mar Abad-Grau

Spain

Pablo Porras Millan

Spain

Paul Spellman

USA

Son Pham

USA

Saurabh Prasad

USA

Dwight Stambolian

USA

Stefan Schulz

Austria

Steffen Sass

Germany

Stephan Pabinger

Austria

Stephane Dray

France

Steven Rozen

Singapore

Stephen Salipante

USA
Vincent Carey

USA

Weiliang Qiu

USA

Subhajit Sengupta

USA

Sunghwan Kim

South Korea

Sukarna Barua

Bangladesh

Zhifu Sun

USA

Sutirtha Chakraborty

USA

Suvendra Kumar Ray India

David Svoboda

Czech Republic

Shi Wang

China

Swapna Mahurkar Joshi

USA

Mikael Benson

Sweden

Sebastian Will

Germany

Frederic Guillaume

Switzerland

Hubert Rehrauer

Switzerland

Federico Santoni

Switzerland

Sylvain Trépout

France

Silke Szymczak

Germany

Bor-Sen Chen

Taiwan

Chung-Feng Liu

Taiwan

Jui-Hung Hung

Taiwan

Kazuhiro Takemoto

Taiwan 


\begin{tabular}{|c|c|c|}
\hline $\begin{array}{l}\text { Akito Taneda } \\
\text { Japan }\end{array}$ & $\begin{array}{l}\text { Tim Beißbarth } \\
\text { Taiwan }\end{array}$ & $\begin{array}{l}\text { Guray Kuzu } \\
\text { Turkey }\end{array}$ \\
\hline $\begin{array}{l}\text { Xiaojia Tang } \\
\text { Japan }\end{array}$ & $\begin{array}{l}\text { German Tischler } \\
\text { Germany }\end{array}$ & $\begin{array}{l}\text { Gabor Tusnady } \\
\text { Turkey }\end{array}$ \\
\hline $\begin{array}{l}\text { Michel Tchuenche } \\
\text { USA }\end{array}$ & $\begin{array}{l}\text { Tiziana Margaria } \\
\text { Germany }\end{array}$ & $\begin{array}{l}\text { Tzachi Hagai } \\
\text { Hungary }\end{array}$ \\
\hline $\begin{array}{l}\text { Tatiana Tatusova } \\
\text { Tanzania }\end{array}$ & $\begin{array}{l}\text { Thomas James Hardcastle } \\
\text { Germany }\end{array}$ & $\begin{array}{l}\text { Uchiyama Ikuo } \\
\text { UK }\end{array}$ \\
\hline $\begin{array}{l}\text { Taylor Wilcox } \\
\text { USA }\end{array}$ & $\begin{array}{l}\text { Tao Liu } \\
\text { UK }\end{array}$ & $\begin{array}{l}\text { Graham Ritchie } \\
\text { Japan }\end{array}$ \\
\hline $\begin{array}{l}\text { Toby Hocking } \\
\text { USA }\end{array}$ & $\begin{array}{l}\text { Tobias Müller } \\
\text { USA }\end{array}$ & $\begin{array}{l}\text { Simon Rogers } \\
\text { UK }\end{array}$ \\
\hline $\begin{array}{l}\text { Tuan Pham } \\
\text { Canada }\end{array}$ & $\begin{array}{l}\text { Tobias Ölschläger } \\
\text { Germany }\end{array}$ & $\begin{array}{l}\text { Neil David Rawlings } \\
\text { UK }\end{array}$ \\
\hline $\begin{array}{l}\text { Ruoqing Zhu } \\
\text { Japan }\end{array}$ & $\begin{array}{l}\text { Kiehl Thomas } \\
\text { Denmark }\end{array}$ & $\begin{array}{l}\text { Magdalena Zarowiecki } \\
\text { UK }\end{array}$ \\
\hline $\begin{array}{l}\text { Teng Zhang } \\
\text { USA }\end{array}$ & $\begin{array}{l}\text { Torben Nielsen } \\
\text { USA }\end{array}$ & $\begin{array}{l}\text { Lachlan Coin } \\
\text { UK }\end{array}$ \\
\hline $\begin{array}{l}\text { Herve Tettelin } \\
\text { USA }\end{array}$ & $\begin{array}{l}\text { Torbjorn Rognes } \\
\text { USA }\end{array}$ & $\begin{array}{l}\text { Makoto Miwa } \\
\text { UK }\end{array}$ \\
\hline $\begin{array}{l}\text { Sissades Tongsima } \\
\text { USA }\end{array}$ & $\begin{array}{l}\text { Torsten Waldminghaus } \\
\text { Norway }\end{array}$ & $\begin{array}{l}\text { Silvia Liverani } \\
\text { UK }\end{array}$ \\
\hline $\begin{array}{l}\text { Tomas Helikar } \\
\text { Thailand }\end{array}$ & $\begin{array}{l}\text { Thomas Pengo } \\
\text { Germany }\end{array}$ & $\begin{array}{l}\text { Vincent Plagnol } \\
\text { UK }\end{array}$ \\
\hline $\begin{array}{l}\text { Theo Niyonsenga } \\
\text { USA }\end{array}$ & $\begin{array}{l}\text { Tim Reviewer } \\
\text { USA }\end{array}$ & $\begin{array}{l}\text { Marion Dawn Teare } \\
\text { UK }\end{array}$ \\
\hline $\begin{array}{l}\text { Thien Ho } \\
\text { Australia }\end{array}$ & $\begin{array}{l}\text { Olivia Reviewer } \\
\text { UK }\end{array}$ & $\begin{array}{l}\text { Roman Laskowski } \\
\text { UK }\end{array}$ \\
\hline $\begin{array}{l}\text { Krishnaraj Thirugnanasambantham } \\
\text { USA }\end{array}$ & $\begin{array}{l}\text { Travis Gagie } \\
\text { UK }\end{array}$ & $\begin{array}{l}\text { Andrea Sottoriva } \\
\text { UK }\end{array}$ \\
\hline $\begin{array}{l}\text { Thomas Faraut } \\
\text { India }\end{array}$ & $\begin{array}{l}\text { Todd Treangen } \\
\text { Finland }\end{array}$ & $\begin{array}{l}\text { David Balding } \\
\text { UK }\end{array}$ \\
\hline $\begin{array}{l}\text { Thomas Lingner } \\
\text { France }\end{array}$ & $\begin{array}{l}\text { William Trimble } \\
\text { USA }\end{array}$ & $\begin{array}{l}\text { Nicholas Furnham } \\
\text { UK }\end{array}$ \\
\hline $\begin{array}{l}\text { Thomas Pfau } \\
\text { Germany }\end{array}$ & $\begin{array}{l}\text { Thomas Rindflesch } \\
\text { USA }\end{array}$ & $\begin{array}{l}\text { Diego Oyarzun } \\
\text { UK }\end{array}$ \\
\hline $\begin{array}{l}\text { Thomas Walter } \\
\text { Luxembourg }\end{array}$ & $\begin{array}{l}\text { Yoshimasa Tsuruoka } \\
\text { USA }\end{array}$ & $\begin{array}{l}\text { Rebecca Cordell } \\
\text { UK }\end{array}$ \\
\hline $\begin{array}{l}\text { Julie Thompson } \\
\text { France }\end{array}$ & $\begin{array}{l}\text { Tom Tullius } \\
\text { UK }\end{array}$ & $\begin{array}{l}\text { Katie Lunnon } \\
\text { UK }\end{array}$ \\
\hline $\begin{array}{l}\text { Tianqi Liu } \\
\text { France }\end{array}$ & $\begin{array}{l}\text { Arzucan Ozgur } \\
\text { USA }\end{array}$ & $\begin{array}{l}\text { Gemma Sharp } \\
\text { UK }\end{array}$ \\
\hline $\begin{array}{l}\text { Tsung-I Lin } \\
\text { USA }\end{array}$ & $\begin{array}{l}\text { Osman Ugur Sezerman } \\
\text { Turkey }\end{array}$ & $\begin{array}{l}\text { Ramon Grima } \\
\text { UK }\end{array}$ \\
\hline
\end{tabular}


Andreas Dr\&Auml;Ger

UK

Bhagwati Gupta

USA

Rasna Walia

USA

Min $\mathrm{He}$

USA

Yaping Liu

USA

Chun-Hsi Huang

USA

Eckart Bindewald

USA

Nathan Clement

USA

Yongchao Liu

USA

Yufeng Wu

USA

Jaques Reifman

USA

Gary Chen

USA

Christopher Hill

USA

Connor Skennerton

USA

Kristin Bennett

USA

Alice Wattam

USA

Tandy Warnow

USA

Swati Biswas

USA

Chen Wang

USA

Peng Yu

USA

Zohreh Talebizadeh

USA

Oncel Tuzel

USA
Moitrayee Bhattacharyya

USA

Chi Zhang

USA

Grzegorz Boratyn

USA

Dima Kozakov

USA

Cesar Ramirez

USA

Yang Dai

USA

Richard Gronostajski

USA

Matthew Nicholson McCall

USA

Ka Yee Yeung

USA

Yuzhen Ye

USA

Brett McKinney

USA

Ann Oberg

USA

Ryan Layer

USA

Hongyi Xin

USA

Giulio Genovese

USA

Po-Ru Loh

USA

Robert Page

USA

Huy Dinh

USA

Patrick Flaherty

USA

Jeremy Goecks

USA

Patricia Chan

USA

William Nes

USA
Stan Pounds

USA

Keith Dunker

USA

Serdar Bozdag

USA

Sawsan Khuri

USA

Yoo-Ah Kim

USA

Ting Chen

USA

Siddhartha Jonnalagadda

USA

Cecilia Arighi

USA

Hagit Shatkay

USA

Anurag Sethi

USA

Justin Guinney

USA

Samuel Payne

USA

Michael Kane

USA

Cong Li

USA

Faheem Mitha

USA

Niina Haiminen

USA

W. Evan Johnson

USA

Qian Sun

USA

Ruilin Liu

USA

Lynette Hirschman

USA

Iosif Vaisman

USA

Radu Herbei

USA 
Volodymyr Melnykov

USA

Donna Karolchilk

USA

Arindam Roychoudhury

USA

Xiaobo Zhou

USA

Yang Li

USA

Yang Yu

USA

David Craig

USA

Qi Li

USA

Yuqing Mao

USA

Zheng Xia

USA

Scott Gerber

USA

Brian Pratt

USA

Laurel Copeland

USA

Zhuohui Gan

USA

Cathy Lawson

USA

Nicole Carnegie

USA

Samuel Deutsch

USA

Nathan Hillson

USA

Rebecca Dickstein

USA

Eitan Halper-Stromberg

USA

Yakir Reshef

USA

Jie Ding

USA
Juhee Lee

USA

Kenneth Cornetta

USA

Libusha Kelly

USA

Louis Sherman

USA

Jun-Tao Guo

USA

Deeptak Verma

USA

Xiaobin Zheng

USA

Xinan Yang

USA

Tong Zhou

USA

Vikas Bansal

USA

Peter Clote

USA

Franz Gruswitz

USA

Diana Delibaltov

USA

Vignesh Jagadeesh

USA

Vesna Memisevic

USA

Seongho Kim

USA

Jianrong Dong

USA

Elena Rivas

USA

Joyee Ghosh

USA

Jiguang Wang

USA

Qin Wang

USA

Rasmi Thomas

USA
Michael Lawrence

USA

Robert Scharpf

USA

Dina Schneidman

USA

Heiko Enderling

USA

Jonas Almeida

USA

Scott Edwards

USA

Youngik Yang

USA

Brian Long

USA

Xinping Cui

USA

Yen-Tsung Huang

USA

Qiong Yang

USA

Hetunandan Kamichetty

USA

Hua Lou

USA

Aiguo Li

USA

Zhenyuan Song

USA

Luis Tari

USA

Dandi Qiao

USA

Mohamed Diwan M Abdulhameed USA

Xueping Yu

USA

Denis Fourches

USA

Robert Kincaid

USA

Graham Snyder

USA 
Gangqing $\mathrm{Hu}$
USA

Nikolaus Schultz

USA

Caitlin Pepperell

USA

Stacey Winham

USA

Bernard Kim

USA

Tarjei Mikkelsen

USA

Matthew Hudson

USA

Surya Saha

USA

Jean-Francois Rual

USA

Ming Wu

USA

Zhenqing Ye

USA

Ying Ding

USA

Benjamin Regner

USA

Beisi Xu

USA

Zhixing Feng

USA

Xuebing Wu

USA

Usman Roshan

USA

Upadhyayula Surya Raghavender USA

Utkarsh Gaur

USA

Vân Anh Huynh-Thu

USA

Valerio Bianchi

USA

Vasu Punj

USA
Vera Van Noort

USA

M. Paola Vera-Licona

USA

A Mohanapriya

USA

Vincent Segura

USA

Vitor Sousa

USA

Veli Mäkinen

USA

Vikas Pejaver

USA

Vsevolod Makeev

USA

Viktoria Szabo

USA

Wolfgang Raffelsberger

USA

Wael Ahmed

USA

Dirk Walther

USA

Qiong Wang

USA

Yinan Wan

USA

Wei Chen

USA

Gang Wu

USA

Wibke Busch

USA

Wim Verhaegh

USA

Wei Jiang

USA

Weizhong Li

USA

William Dampier

USA

William Pearson

USA
Mengyun Wu

USA

Cen Wu

USA

Xiangqin Cui

Israel

Rui Xia

USA

Xiaoming Wang

Belgium

Xiaoqing Yu

Italy

Xiaowei Zhou

USA

Xiaoxiao Liu

Belgium

Xiaxia Yu

USA

Xin Wang

USA

Xing Chen

India

Xinghua Lu

France

Xingyu Li

Switzerland

Xiting Yan

Finland

Xiu Huang

USA

Xiaodong Liu

Russia

Xueqiu Jian

Spain

Jianpeng $\mathrm{Xu}$

France

Xujing Wang

Egypt

Xiang Wan

Germany

Xinan Yang

USA

Yanay Ofran

USA 


$\begin{array}{lll}\text { Yang Du } & \text { Yun Xu } & \text { Zhen Xie } \\ \text { USA } & \text { Canada } & \text { USA } \\ \text { Yanni Sun } & \text { Yupeng Cun } & \text { Zheyang Wu } \\ \text { USA } & \text { USA } & \text { USA } \\ \text { Yao Fu } & \text { Yuping Zhang } & \text { Fangqing Zhao } \\ \text { USA } & \text { USA } & \text { China } \\ \text { Yarden Katz } & \text { Yuwen Liu } & \text { Zhi Zhou } \\ \text { Germany } & \text { USA } & \text { China } \\ \text { Yu Fan } & \text { Ioannis Vlachos } & \text { Zhizhuo Zhang } \\ \text { Netherlands } & \text { USA } & \text { USA } \\ \text { Yon Hui Sarah Kim } & \text { Yingying Wei } & \text { Zhou Xiaoyuan } \\ \text { Hong Kong } & \text { Hong Kong } & \text { Israel } \\ \text { Yin Liu } & \text { Yoav Broza } & \text { Bin Zhu } \\ \text { USA } & \text { China } & \text { Germany } \\ \text { Zhao Yinjun } & \text { Yate-Ching Yuan } & \text { Ziding Zhang } \\ \text { USA } & \text { USA } & \text { USA } \\ \text { Yoji Nakamura } & \text { Zachary Charlop-Powers } & \text { Urs Ziegler } \\ \text { USA } & \text { USA } & \text { USA } \\ \text { Yasset Perez-Riverol } & \text { Osvaldo Zagordi } & \text { Sajjad Karim } \\ \text { China } & \text { USA } & \text { USA } \\ \text { Yao Shen } & \text { Wei Zhang } & \text { Shihua Zhang } \\ \text { USA } & \text { USA } & \text { USA } \\ \text { Yuan Jiang } & \text { Yi Zhao } & \\ \text { USA } & \text { USA } & \\ \text { Yunfei Li } & \text { Zhengqing Ouyang } & \\ \text { USA } & \text { USA } & \end{array}$

